\title{
27-Day Modulation of Cosmic Ray Intensities During the Last Two Solar Minima
}

\author{
R. A. Leske ${ }^{* a}$, A. C. Cummings ${ }^{a}$, R. A. Mewaldt ${ }^{a}$, E. C. Stone ${ }^{a}$, T. T. von \\ Rosenvinge $^{b}$, and M. E. Wiedenbeck ${ }^{c}$ \\ ${ }^{a}$ California Institute of Technology, Pasadena, CA 91125 USA \\ ${ }^{b}$ NASA/Goddard Space Flight Center, Greenbelt, MD 20771 USA \\ c Jet Propulsion Laboratory, California Institute of Technology, Pasadena, CA 91109 USA \\ E-mail: ralesrl.caltech.edu, ace@srl.caltech.edu, \\ rmewaldtesrl.caltech.edu, ecs@srl.caltech.edu, tycho@starpower. net, \\ Mark.E.Wiedenbeck@jpl.nasa.gov
}

\begin{abstract}
Variations in both anomalous cosmic ray (ACR) and galactic cosmic ray (GCR) intensities at the solar rotation period ( $\sim 27$ days) are often observed, and appear throughout much of 2016 into 2019 during the present $\mathrm{A}>0$ polarity solar minimum. During the previous $\mathrm{A}<0$ solar minimum, such variations were evident at energies from a few $\mathrm{MeV}$ to many $\mathrm{GeV}$ from mid-2007 through late 2008, and were much larger for ACRs than for GCRs at comparable energy. The particle intensities had an inverse power-law correlation with the solar wind speed, intensity peaks occurred only near alternate crossings of the heliospheric current sheet, and the variations in amplitudes were larger than typical for an $\mathrm{A}<0$ epoch. The heliospheric environment was unusual compared with earlier cycles, with lower solar wind dynamic pressure, weaker interplanetary magnetic field strength, and record-setting GCR intensities. The present high-energy GCR intensities are higher than ever before reported in an $\mathrm{A}>0$ cycle (although lower than in several $\mathrm{A}<0$ cycles), indicating that unusual modulation conditions still prevail.

Using high statistical precision measurements from the ACE spacecraft along with neutron monitor data, we present observations of the 27-day intensity variations in both ACRs and GCRs during the last two solar minima and describe how they depend on particle energy, rigidity, and spectrum, and on interplanetary conditions such as solar wind speed, magnetic field strength, and current sheet tilt. We compare the variations observed in this cycle with those in the previous opposite polarity cycle and discuss implications for particle transport in the heliosphere.
\end{abstract}

36th International Cosmic Ray Conference -ICRC2019-

July 24th - August 1st, 2019

Madison, WI, U.S.A.

*Speaker. 


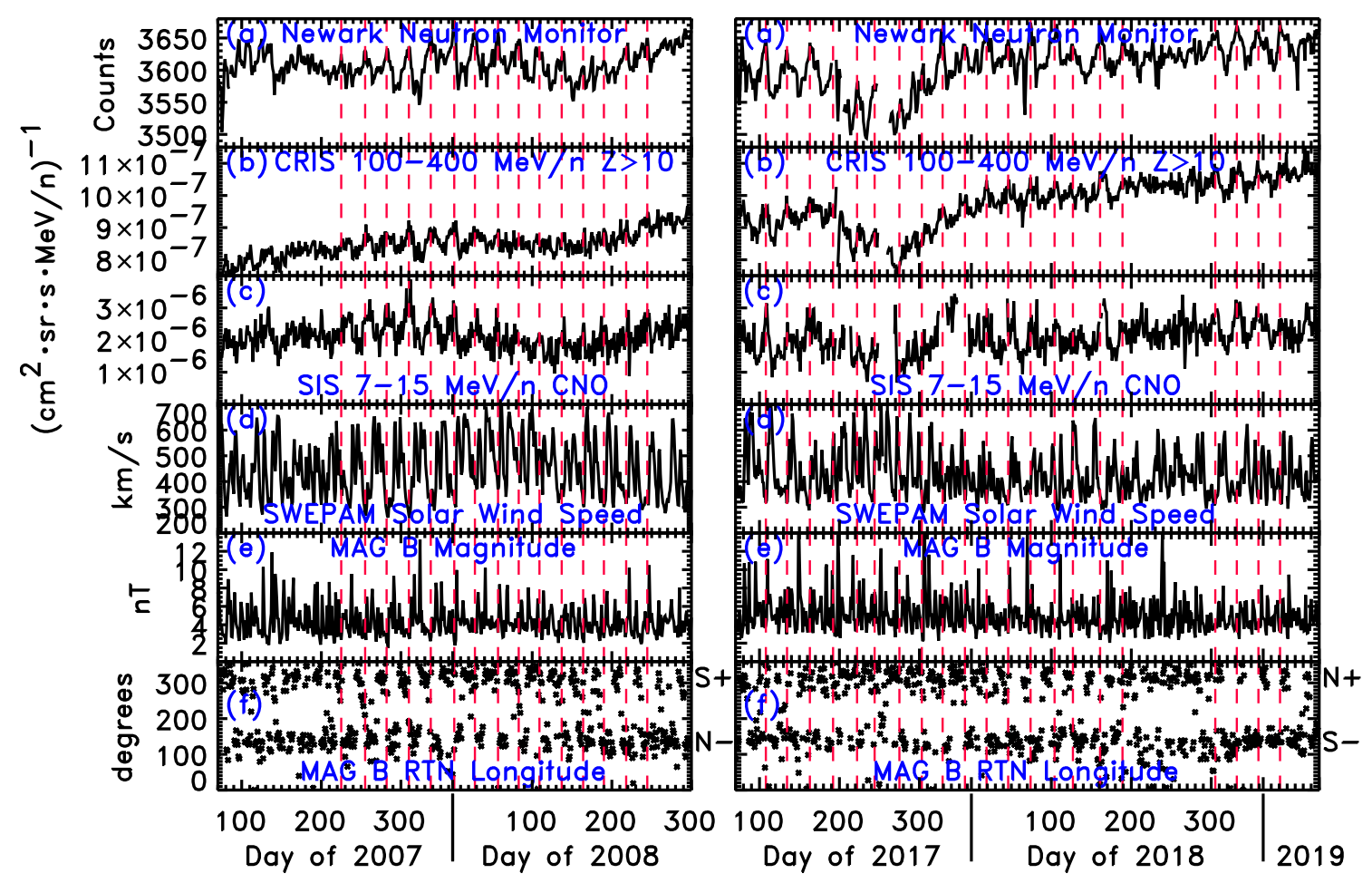

Figure 1: Daily-averaged values of: $(a)$ the count rate of the Newark neutron monitor, $(b)$ GCRs from ACE/CRIS, $(c)$ mostly ACRs from ACE/SIS, $(d)$ the solar wind speed from ACE/SWEPAM, $(e)$ the magnitude of the magnetic field from ACE/MAG, and $(f)$ the magnetic field RTN longitude (i.e., $\tan ^{-1}\left(B_{T} / B_{R}\right)$ ) from ACE/MAG, for a period with pronounced 27-day variations during the last solar minimum (left; [1]) and the present solar minimum (right). Red vertical lines mark peaks in panel (a) spaced by 27 days.

\section{Introduction}

For over 80 years it has been known that quiet-time cosmic ray intensities often vary at the solar rotation period ( $\sim 27$ days synodic, or $\sim 26$ days sidereal period) [2]. Such variations have been observed in many species and energies, in both galactic cosmic rays (GCRs) and anomalous cosmic rays (ACRs) at energies ranging from several $\mathrm{MeV} /$ nucleon to many $\mathrm{GeV} /$ nucleon [3], at heliospheric locations inside of $1 \mathrm{AU}$ [4] to far beyond $60 \mathrm{AU}$ [5], and from the ecliptic plane to high latitudes ([6] and references therein). (See [7] for a thorough review of this topic). Studies of these variations may reveal details of particle transport and modulation on a variety of distance scales within the heliosphere.

The last two solar minima have exhibited unusually low levels of GCR modulation. In the 2009 A $<0$ minimum, weak interplanetary magnetic fields with low levels of magnetic turbulence [8] and reduced solar wind dynamic pressure [9] resulted in record-setting GCR intensities [10], while in the present minimum neutron monitor rates are higher than in any previously measured A $>0$ cycle [11]. Both these minima had periods with pronounced 27-day variations: from mid2007 through mid-2008 in the previous minimum [1], and from mid-2017 through early 2019 in the present minimum, as discussed here, and as shown in Figure 1. (Although much larger amplitude variations in GCR intensities were observed in 2014-2015 [12], this occurred near solar maximum 


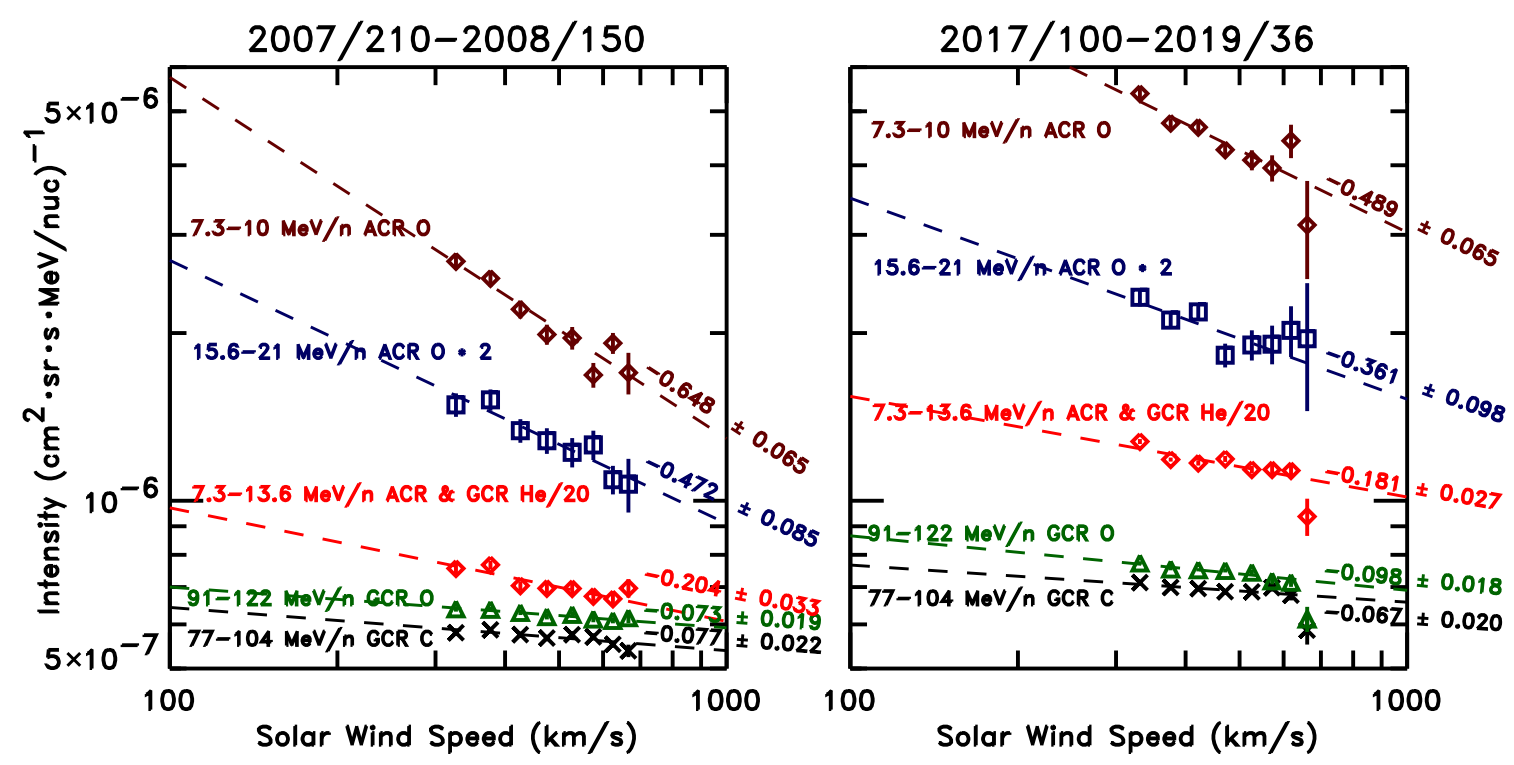

Figure 2: Intensities of the ACR and GCR species indicated versus the binned solar wind speed during the periods 2007/210-2008/150 (left) and 2017/100-2019/36 (right). Dashed lines show power-law fits, with indices as labeled.

when ACR intensities had not yet recovered, so we do not include this period in our study).

\section{Observations}

The 27-day variations, when present, appear in both GCRs and ACRs across a wide range of energies. Figure 1 shows similarities in the time series of neutron monitor rates, lower-energy GCR measurements from CRIS on ACE, and ACR rates from ACE/SIS during both the previous and present solar minima. Peaks in the energetic particle rates appear at or near minima in the solar wind speed, which often occur near magnetic sector crossings since the heliospheric current sheet (HCS) is embedded in the slow-speed wind. Generally, however, only every other sector crossing is associated with an intensity peak, as reported by [3] and discussed in more detail in section 3 below.

In [1], we detrended the data by subtracting 27 -day running averages from the daily averages to remove the longer-term variations in solar modulation. We compared the detrended Newark neutron monitor data with the detrended solar wind speed, density, dynamic pressure, magnetic field strength, and rms variation of the magnetic field, and found the best correlation with the solar wind speed, as in earlier studies by others [4].

Binning the ACE GCR and ACR data in $50 \mathrm{~km} / \mathrm{s}$ solar wind speed bins (using solar wind speeds from ACE/SWEPAM) reveals that the energetic particle intensities vary inversely with solar wind speed as power laws (Figure 2), with steeper slopes (and thus greater amplitudes of the 27day variations) for the ACRs than the GCRs. Although the slopes tend to be slightly shallower in the present $\mathrm{A}>0$ solar minimum compared with the previous $\mathrm{A}<0$ solar minimum, the largest difference in Figure 2 is for 7.3-10 MeV/nucleon ACR oxygen, and here the slopes differ by only $\sim 1.7 \sigma$. The slopes (and amplitudes of the 27-day variations) become smaller for ACRs at higher 


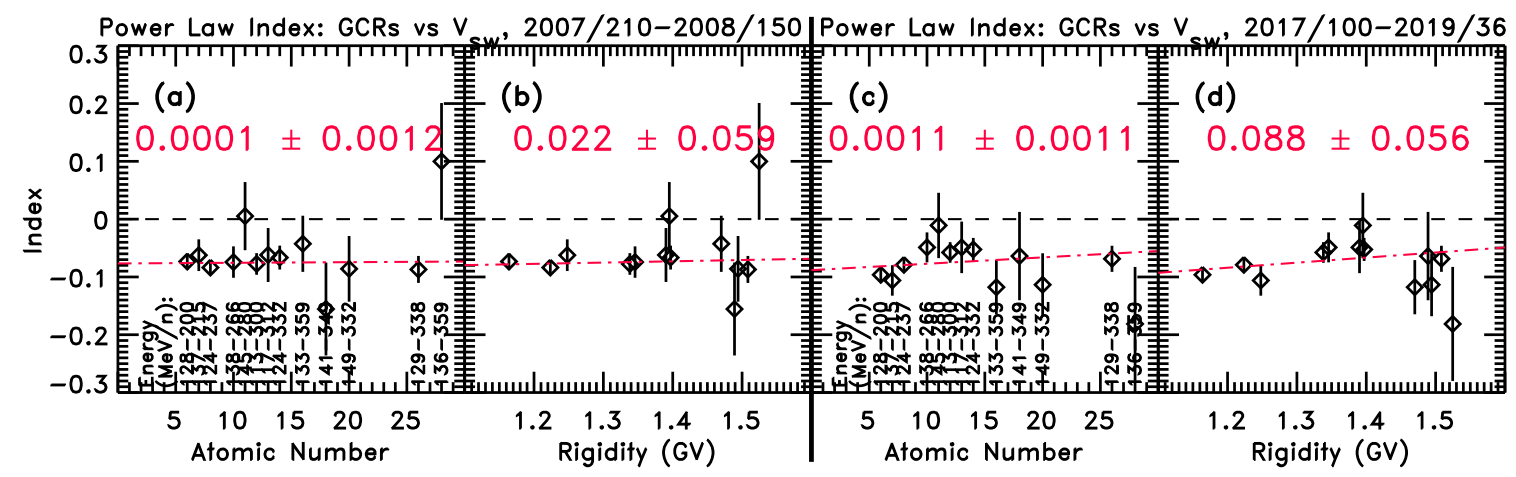

Figure 3: Indices of the power-law fits of the intensity of ACE/CRIS GCRs versus solar wind speed (see Figure 2) as a function of atomic number (panels $(a)$ and $(c)$ ) at the energies indicated, or rigidity (panels (b) and $(d)$ ), for the previous solar minimum (panels $(a)$ and $(b)$ ) and the present minimum (panels $(c)$ and $(d)$ ). Linear fits, their slopes, and uncertainties, are shown in (red).

energies (as shown in more detail below in Figure 4) or for species such as He that are a mixture of both ACRs and GCRs at these energies [13].

Using the high-statistics dataset made possible by ACE/CRIS, we examine the variations of the GCRs for 13 different elements in Figure 3. All these elements have very nearly the same powerlaw index when plotted vs solar wind speed, at least in the energy interval measured by ACE/CRIS. There is essentially no significant dependence on either element or rigidity in CRIS (the variation in rigidity differs from perfectly flat by only $\sim 1.6 \sigma$ in the recent solar minimum, and this is the strongest dependence in Figure 3). The average of these indices in the previous solar minimum is $-0.075 \pm 0.006$, and in the present solar minimum is $-0.077 \pm 0.006$, so the GCR intensity variation with solar wind speed does not seem to have changed from the last $\mathrm{A}<0$ minimum to the present $\mathrm{A}>0$ minimum. The overall average index across both minima is $-0.076 \pm 0.004$, which represents an intensity variation of $5.4 \pm 0.3 \%$ for a factor of two change in the solar wind speed.

Changes in the energy spectra between high and low solar wind speeds are illustrated in Figure 4. Within statistical fluctuations, above $\sim 100 \mathrm{MeV} /$ nucleon the intensities of GCR N, O, and Ne are $\sim 5 \%$ higher in the slow wind than during the high-speed streams, as expected from the indices presented and discussed in Figure 3. ACR N, O, and Ne, however, all have larger amplitude 27-day variations that grow with decreasing energy to a value of $\sim 50-60 \%$ in the $2007-2008$ solar minimum and to $\sim 30 \%$ in the present minimum. Thus, although the amplitude of the GCR variations is similar during the past two minima, that of the ACR variations is substantially less now than in the previous minimum.

\section{Discussion}

In our earlier work [1], we found that the energy dependence in the ACR amplitudes can be empirically reproduced by mixing of high-amplitude ACRs with low-amplitude GCRs. There we used the carbon spectrum to estimate the amount of GCR background mixed with the ACR oxygen. Assuming both ACR O and GCR O have energy-independent amplitudes (of 55\% and $5 \%$, respectively), simple mixing of the two results in the solid curve in the lower left panel of Figure 4, which provides a reasonable match to the data. 


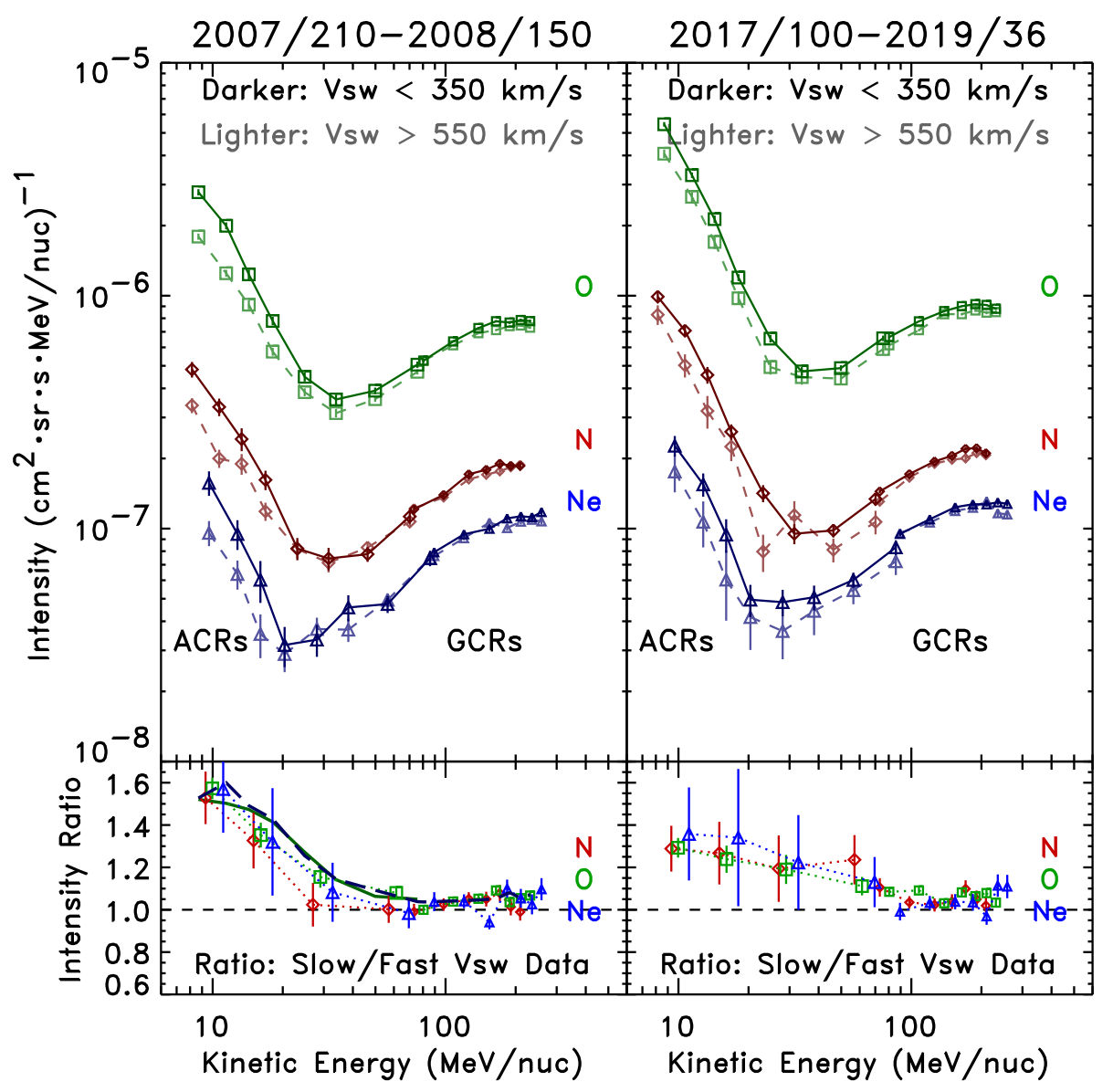

Figure 4: Energy spectra (top panels) from ACE/SIS (8 lowest energy points, larger symbols) and ACE/CRIS (7 highest energy points, smaller symbols) for O (squares), $\mathrm{N}$ (diamonds), and $\mathrm{Ne}$ (triangles) during the previous solar minimum period (left) and present solar minimum (right). For each element and time interval two spectra are shown, one for periods when the solar wind speed was $>550 \mathrm{~km} / \mathrm{s}$ (lighter dashed curves) and one when the speed was $<350 \mathrm{~km} / \mathrm{s}$ (darker solid curves). (The average solar wind speeds in these two speed intervals were 613 and $316 \mathrm{~km} / \mathrm{s}$ during the first period, and 593 and $326 \mathrm{~km} / \mathrm{s}$ during the second). Ratios of the spectra at the two solar wind speeds are plotted in the bottom panels. The solid curve in the lower left panel illustrates the energy dependence expected just due to mixing of ACR and GCRs, while the thick dashed curve uses the formula from $[14,15]$ as described in the text.

Using a force field modulation model, the energy dependence of the fractional change in GCR and ACR counting rates, $c$, is given by $\Delta c / c=-3 C \Delta \phi / \beta K(P)$, where $\beta$ the particle velocity, $K(P)$ the diffusion coefficient as a function of rigidity, $\Delta \phi$ the change in the modulation parameter, and $C$ the Compton-Getting factor $[14,15]$. If the particle spectrum is a power-law in kinetic energy $E$ with index $\gamma$, then $C=(2-\alpha \gamma) / 3$, where $\alpha=\left(E+2 E_{0}\right) /\left(E+E_{0}\right)$ where $E_{0}$ is the rest mass energy. Taking $K$ proportional to $P^{0.5}$ as in [14], evaluating these formulae, and normalizing to 10 $\mathrm{MeV} /$ nucleon ACR oxygen results in the dashed curve in the lower left panel of Figure 4, which is essentially the same as the simple mixing curve derived above. These formulae yield a small mass-dependence for the amplitude of the ACR intensity variations which is not inconsistent with the large statistical uncertainties of our data. (As we reported in [1], if the amplitude for ACR O is 

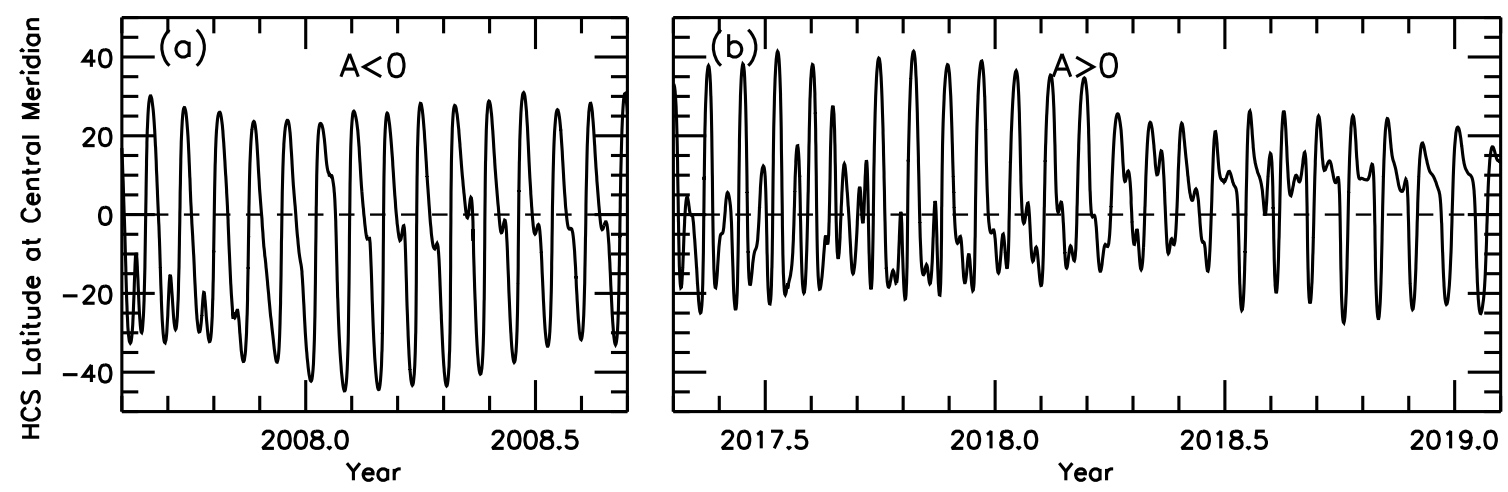

Figure 5: HCS latitude in the corona at central meridian as a function of time (a) during the $2008 \mathrm{~A}<0$ solar minimum and $(b)$ during the present $\mathrm{A}>0$ solar minimum, using coronal field map data from the Wilcox Solar Observatory.

$55 \%$, the predicted amplitudes for $\mathrm{N}$ and $\mathrm{Ne}$ would be $59 \%$ and $49 \%$ respectively).

The underlying contributions to the 27-day variations are not entirely clear. Drift effects create a latitudinal intensity gradient about the HCS in both ACRs and GCRs [16], which might be expected to produce intensity variations as the tilted current sheet rotates past an observer $[17,18]$. Indeed, significant correlations between the local latitudinal gradient and the amplitude of the 27day variations have been reported [19], but since intensity variations often show a 27-day period even when the HCS is crossed twice per solar rotation, more than just the latitudinal gradient must be involved. Some studies using high time resolution find that quiet-time cosmic ray intensity decreases coincide with stream interface crossings and appear unrelated to sector boundaries [7, 20], while others employing superposed epoch analysis over many years report that cosmic ray intensities peak at sector boundaries [21, 22]. For both the 1975 and $1996 \mathrm{~A}>0$ solar minima, Reames and $\mathrm{Ng}$ [3] found peak cosmic ray intensities at north-to-south (outward-to-inward polarity) HCS crossings with minima at south-to-north crossings, while the phase in the intervening $\mathrm{A}<0$ minimum was unclear. As shown in panels $(f)$ of Figure 1, in the 2007-2008 period we find that peaks appear at outward-to-inward heliomagnetic polarity HCS crossings, which represent south-to-north crossings heliographically during this $\mathrm{A}<0$ interval. Curiously, during the present $\mathrm{A}>0$ minimum, the phasing of intensity peaks with sector crossings seems to transition from outward-to-inward (north-to-south) in late 2017 to inward-to-outward (south-to-north) by early 2019, suggesting that the polarity or direction of the HCS crossing is not fundamentally important to the phasing of the variations.

We have previously noted [1] that the shape of the HCS is usually asymmetric, resulting in a more abrupt change in heliomagnetic latitude when crossing the sheet in one direction and a slower transition in the other (Figure 5). In 2007-2008 south-to-north HCS crossings were more gradual, keeping the HCS near the ecliptic longer than during the more rapid north-to-south crossings. During $\mathrm{A}<0$ particles drift inward along the sheet, and the solar wind speed and therefore outward convection is lower at the sheet, so the gradual HCS crossings should favor higher particle intensities, as observed. However, it was the shorter, abrupt HCS crossings that seemed to be favored during the $1996 \mathrm{~A}>0$ minimum in the Reames and $\mathrm{Ng}$ [3] study, while the pattern in the present minimum is less clear, particularly in late 2018, when there was not much 
difference in the abruptness of the crossings (Figure 5). Perusal of Hinode XRT images (at solar.physics.montana.edu/HINODE/XRT/) seems to suggest that the dominant polar coronal hole was in the south in 2008 , but in the north in late 2017 , with some growth in the southern hole by late 2018. It seems likely that the key factor in determining which of the two HCS crossings per rotation leads to an increase in particles, and which to a decrease, is simply a question of whether the observer is crossing out of the hemisphere with the dominant coronal hole (and thus into slower solar wind and higher particle intensities) or into it.

\section{Acknowledgments}

This work was supported by NASA under grant 80NSSC18K0223. We thank the ACE/SWEPAM and MAG instrument teams and the ACE Science Center (http://www.srl.caltech.edu/ACE/ ASC) for making their solar wind and magnetic field data publicly available. We acknowledge the NMDB database (www.nmdb.eu), founded under the European Union's FP7 program (contract 213007) for hosting and distributing the Newark neutron monitor data from the Bartol Research Institute, supported by the NSF under grants ANT-0739620 and ANT-0838839. We thank Todd Hoeksema and the Wilcox Solar Observatory (http://wso.stanford.edu) for providing the HCS and coronal field map data.

\section{References}

[1] R. A. Leske, A. C. Cummings, R. A. Mewaldt, E. C. Stone, T. T. von Rosenvinge, and M. E. Wiedenbeck, Observed 27-Day Variations in Cosmic Ray Intensities During the Cycle 23/24 Solar Minimum, Proc. 32nd Internat. Cosmic Ray Conf. (Beijing) 11 (2011) 194-197.

[2] S. E. Forbush, On cosmic-ray effects associated with magnetic storms, Terrestrial Magnetism and Atmospheric Electricity (Journal of Geophysical Research) 43 (1938) 203.

[3] D. V. Reames and C. K. Ng, On the Phase of the 27 Day Modulation of Anomalous and Galactic Cosmic Rays at 1 AU during Solar Minimum, Astrophys. J. Lett. 563 (2001) L179-L182.

[4] I. G. Richardson, G. Wibberenz, and H. V. Cane, The relationship between recurring cosmic ray depressions and corotating solar wind streams at $\leq 1$ AU: IMP 8 and Helios 1 and 2 anticoincidence guard rate observations, J. Geophys. Res. 101 (1996) 13483-13496.

[5] R. Decker, S. M. Krimigis, A. G. Ananth, D. C. Hamilton, and M. E. Hill, Small-Scale Variations in ACR Intensities at Voyagers 1 and 2 in 1992-1998, Proc. 26th Internat. Cosmic Ray Conf. (Salt Lake City) 7 (1999) 512.

[6] R. B. McKibben, Three-dimensional Solar Modulation of Cosmic Ray and Anomalous Components in the Inner Heliosphere, Space Sci. Rev. 83 (1998) 21-32.

[7] I. G. Richardson, Energetic Particles and Corotating Interaction Regions in the Solar Wind, Space Sci. Rev. 111 (2004) 267-376.

[8] E. J. Smith and A. Balogh, Decrease in heliospheric magnetic flux in this solar minimum: Recent Ulysses magnetic field observations, Geophys. Res. Lett. 35 (2008) L22103.

[9] D. J. McComas, R. W. Ebert, H. A. Elliott, B. E. Goldstein, J. T. Gosling, N. A. Schwadron, and R. M. Skoug, Weaker solar wind from the polar coronal holes and the whole Sun, Geophys. Res. Lett. 35 (2008) L18103. 
[10] R. A. Mewaldt, A. J. Davis, K. A. Lave, R. A. Leske, E. C. Stone, M. E. Wiedenbeck, W. R. Binns, E. R. Christian, A. C. Cummings, G. A. de Nolfo, M. H. Israel, A. W. Labrador, and T. T. von Rosenvinge, Record-setting Cosmic-ray Intensities in 2009 and 2010, Astrophys. J. Lett. 723 (2010) L1-L6.

[11] R. A. Leske, A. C. Cummings, R. A. Mewaldt, C. Cohen, E. C. Stone, and M. E. Wiedenbeck, Anomalous and Galactic Cosmic Ray Intensities at 1 AU During the Approach to the Cycle 24/25 Solar Minimum and Throughout the Last 20 Years, AGU Fall Meeting Abstracts (2017).

[12] A. Gil and K. Mursula, Comparing Two Intervals of Exceptionally Strong Solar Rotation Recurrence of Galactic Cosmic Rays, J. Geophys. Res. 123 (2018) 6148-6160.

[13] G. A. de Nolfo, W. R. Binns, C. M. S. Cohen, A. C. Cummings, A. J. Davis, J. S. George, P. L. Hink, M. H. Israel, K. Lave, R. A. Leske, R. A. Mewaldt, I. V. Moskalenko, R. C. Ogliore, E. C. Stone, T. T. von Rosenvinge, M. E. Wiedenbeck, and N. E. Yanasak, Cosmic-ray helium intensities over the solar cycle from ACE, Proc. 30th Internat. Cosmic Ray Conf. (Mérida) 1 (2008) 813-816.

[14] T. T. von Rosenvinge and C. Paizis, Amplitudes of solar modulation of low energy cosmic rays, Proc. 17th Internat. Cosmic Ray Conf. (Paris) 10 (1981) 69-72.

[15] C. Paizis, B. Heber, P. Ferrando, A. Raviart, B. Falconi, S. Marzolla, M. S. Potgieter, V. Bothmer, H. Kunow, R. Müller-Mellin, and A. Posner, Amplitude evolution and rigidity dependence of the 26-day recurrent cosmic ray decreases: COSPIN/KET results, J. Geophys. Res. 104 (1999) 28241-28248.

[16] J. R. Jokipii and B. Thomas, Effects of drift on the transport of cosmic rays. IV - Modulation by a wavy interplanetary current sheet, Astrophys. J. 243 (1981) 1115-1122.

[17] G. Newkirk, Jr. and L. A. Fisk, Variation of cosmic rays and solar wind properties with respect to the heliospheric current sheet. I - Five-GeV protons and solar wind speed, J. Geophys. Res. 90 (1985) 3391-3414.

[18] J. R. Jokipii and J. Kóta, Three-Dimensional Cosmic-Ray Simulations: Heliographic Latitude and Current-Sheet Tilt, Space Sci. Rev. 72 (1995) 379-384.

[19] M. Zhang, A Linear Relationship between the Latitude Gradient and 26 Day Recurrent Variation in the Fluxes of Galactic Cosmic Rays and Anomalous Nuclear Components. I. Observations, Astrophys. J. 488 (1997) 841-853.

[20] K. Ghanbari, V. Florinski, X. Guo, Q. Hu, and R. Leske, Galactic Cosmic Rays Modulation in the Vicinity of Corotating Interaction Regions: Observations During the Last Two Solar Minima, Astrophys. J. (to be submitted, 2019).

[21] K. Fujimoto, H. Kojima, K. Murakami, and K. Nagashima, IMF sector boundary and cosmic ray intensity variations, Proc. 17th Internat. Cosmic Ray Conf. (Paris) 4 (1981) 72-75.

[22] Badruddin, R. S. Yadav, and N. R. Yadav, Intensity variation of cosmic rays near the heliospheric current sheet, Planet. Space Sci. 33 (1985) 191-201. 